\title{
Interactive comment on "New evidence for atmospheric mercury transformations in the marine boundary layer" by Ben Yu et al.
}

\section{Ben Yu et al.}

yuben09@mails.ucas.ac.cn

Received and published: 4 June 2020

In this study, the authors present a comprehensive study on the isotope compositions of atmospheric total gaseous mercury (TGM) and particulate bound mercury (PBM) in the marine boundary layer over Chinese seas. As I know, this is a first study for investigating the isotope compositions of atmospheric $\mathrm{Hg}$ species and their underling mechanisms in MBL. It is therefore novel and would contribute significantly the $\mathrm{Hg}$ isotope research field. The manuscript is overall well written and organized. I agree with the interpretations on the mechanisms associated with the variations of $\mathrm{Hg}$ isotope compositions. Actually, I have revised this manuscript when it was submitted to another journal. I found the authors have address most of my and other reviewer's comments properly, and the paper is currently in a good quality. I would therefore suggest a 
publication of the manuscript in ACP with minor revisions.

Response: We appreciate very much the valuable comments from you and the other ACPD previous reviewers. These comments really improved our manuscript. We hope this study could provide useful data and discussion for better understanding the atmospheric $\mathrm{Hg}$ transformation and transport, especially in marine boundary layer.

line 15: should be 'isotopic compositions of total gaseous mercury (TGM) and particulate bound mercury (PBM) collected. . .' line 18: should be 'isotopic compositions of TGM in MBL were affected. ..'

Response: Thank you very much for these linguistic modifications. These two modifications have been made in the revised manuscript.

Line 19-20: the statement of "lower air temperature could promote the positive..." is not clear. I would suggest to specify to "TGM D199Hg values were significantly positively correlated with air temperature...', and then the author may interpret the potential mechanisms.

Response: The sentence has been changed to 'D199Hg values of TGM were significantly positively correlated with air temperature in summer, indicating that processes inducing positive odd-MIF in TGM could be more active at low temperatures, while the relative processes might be weak in winter.'

Section 2.1: I would suggest to add a figure to show the cruise and sampling locations of the isotope samples in the main manuscript.

Response: We thank the referee for this suggestion. Indeed, we made three GIF files to show the cruise and wind field during three sampling cruises, respectively. We have uploaded the GIF files to https://doi.org/10.5281/zenodo.3871222 as supporting information. The announcement of these supporting information has been added in the Data Availability part in the revised manuscript.

Line 150-154: the diagnostics of using $\mathrm{D} 199 \mathrm{Hg} / \mathrm{d} 202 \mathrm{Hg}$ ratios is great. But I think it 
should simply introduce the ratios obtained from previous laboratory studies or source materials, and this would help you to figure out the major factors.

Response: We agree with the referee. Description on this ratios and regression slopes reported in references have been added as 'This fitted curve always indicated a mixing of plumes with different isotopic fingerprints (Demers et al., 2015;Yu et al., 2016;Fu et al., 2018). Especially a $\sim-0.1$ slope could be shaped when mixing of plumes from anthropogenic emissions characterized by negative $\delta 202 \mathrm{Hg}$ and near-zero $\Delta 199 \mathrm{Hg}$ values, and plumes from remote areas characterized by positive $\delta 202 \mathrm{Hg}$ and negative $\Delta 199 \mathrm{Hg}$ values, e.g., three slopes of $-0.09,-0.13$, and -0.07 observed in TGM from Mt. Damei, Mt. Ailao, and Beijing, China, respectively (Yu et al., 2016), and -0.095 observed in TGM/GEM and source materials worldwide (Fu et al., 2018)'

Line 168: 'TGM collected in two summer cruises ... ranges' should be 'TGM collected in two summer cruises were characterized by significantly negative $\mathrm{d} 202 \mathrm{Hg}$ and nearzero D199Hg values. Line 177-179: 'the back-trajectory ... in TGM' should reword as 'Backward trajectory analysis showed that higher $\mathrm{D} 199 \mathrm{Hg}$ values were associated with air masses originated from both mainland China and open oceans'

Response: We thank the reviewer for these comments. The modifications have been made in the revised manuscript.

Line 202-205: need to be rewritten. Note that the MIF signatures of gaseous $\mathrm{Hg}$ (II) emitted from anthropogenic sources and produced via atmospheric oxidation should be different. The former case would have near-zero value, while the latter case would have significantly positive value according to precipitation observations. These two should be differentiated here.

Response: Here we use the $\mathrm{D} 200 \mathrm{Hg}$ in samples to evaluate the contribution from wet precipitation to TGM and PBM in this study. This method was also used in publications. For instance, Enrico et al. (2016), DOI: 10.1021/acs.est.5b06058, evaluated the contributions from wet precipitation to the $\mathrm{Hg}$ deposited in peat bogs. Higher $\mathrm{D} 200 \mathrm{Hg}$ 
values ( $\sim 0.2 \%$ o were observed in wet precipitation worldwide, no matter the study sites were in urban/rural or remote areas, with or without the impact from anthropogenic emissions. Therefore the observed near-zero $\mathrm{D} 200 \mathrm{Hg}$ values in TGM and PBM in this study suggested limited contributions from $\mathrm{Hg}(\mathrm{II})$ in wet precipitations via photoreduction and re-emission from droplet surfaces. We have updated these sentences to more accurate description on that.

Line 210-215: the D199Hg/D201Hg ratio for PBM should be presented.

Response: Ratio data $(6.8 \pm 8.4,1 \mathrm{SD}, \mathrm{n}=9)$ has been added in the revised manuscript.

Line 285-293: I agree with your interpretation that oceanic GEM emission should play a minor role. However, as the isotopic composition of oceanic GEM emissions have not been well constrained, I would suggest to soft these statements. Maybe this process could be completely excluded. Zheng et al., investigated the MDF of $\mathrm{Hg}$ isotope during aqueous $\mathrm{Hg}(0)$ evaporation. Note that GEM emission from water is driven by evaporation of $\mathrm{Hg}(0)$ from water (the isotopic compositions are unknown) and wateratmosphere interface photoreduction (the isotopic compositions of initial water are unknown). So it is difficult to known whether this source generate positive or negative MIF.

Response: We agree with the referee that isotopic compositions in dissolved gaseous $\mathrm{Hg}$ in surface sea water are unknown. We have highlighted the unknown fact in the revised manuscript and have deleted the arbitrary discussion in this part.

Interactive comment on Atmos. Chem. Phys. Discuss., https://doi.org/10.5194/acp-2020-397, 2020. 